\title{
Role of intraoperative arthrogram in decision making of closed versus medial open reduction of developmental hip dysplasia
}

\author{
Amin A. Ahmed*, Mohie El Din Fadel
}

Department of Orthopaedic Surgery and Traumatology, Faculty of Medicine, Alexandria University, Egypt

Received: 09 August 2019

Revised: 22 August 2019

Accepted: 27 August 2019

\section{*Correspondence: \\ Dr. Amin A. Ahmed, \\ E-mail: aminrazek@yahoo.com}

Copyright: () the author(s), publisher and licensee Medip Academy. This is an open-access article distributed under the terms of the Creative Commons Attribution Non-Commercial License, which permits unrestricted non-commercial use, distribution, and reproduction in any medium, provided the original work is properly cited.

\begin{abstract}
Background: Hip arthrography in developmental dysplasia of the hip (DDH) has the advantages of viewing the shape and size of the cartilaginous part of both the femoral head and acetabulum, besides the soft tissue obstacles. The aim of the work was to assess the role of arthrogram in evaluation of closed reduction of developmental dysplasia of the hip.

Methods: This prospective study included 30 patients with 36 involved hips who presented with DDH. This study included 7 males $(23.3 \%)$ and 23 females $(76.7 \%)$. There were 6 patients with bilateral hip involvement $(20.0 \%)$ and 24 patients with unilateral involvement (80.0\%). In this study we used $3 \mathrm{ml}$ Urografin $76 \%$ diluted in $7 \mathrm{ml}$ saline and 3 to $5 \mathrm{ml}$ of diluted Urografin was injected into the hip joint.

Results: Out of the 36 hips, closed reduction was successful in 33 hips (91.7\%) and failed in 3 (8.3\%). After the interpretation of the arthrogram we considered the reduction of $7(21.2 \%)$ reduced hips nonconcentric because of the soft tissue obstacles. In the 7 hips with nonconcentric reduction besides the 3 hips with failed closed reduction, we proceeded to open reduction of the hip through medial approach. At the end of follow up, all hips (100\%) showed concentric reduction without re-dislocation in any case.

Conclusions: Hip arthrogram is a very reliable method in diagnosing hip concentricity and the presence of soft tissue obstacles in management of DDH by closed reduction. Arthrogram helps the operator to decide open reduction in such cases achieving excellent results in all cases.
\end{abstract}

Keywords: Developmental hip dysplasia, Arthrogram, Closed reduction, Medial approach

\section{INTRODUCTION}

Developmental dysplasia of the hip (DDH) includes femoral head subluxation or dislocation and/or acetabular dysplasia. ${ }^{1}$ Most notably, Ortolani, an Italian pediatrician in the early 1900s, evaluated, diagnosed, and began treating hip dysplasia. ${ }^{2}$ The main objective of closed reduction in $\mathrm{DDH}$ is to attain and maintain concentric reduction without the occurrence of avascular necrosis (AVN) while the hip remodels. ${ }^{3}$ It is generally accepted that a successful outcome of closed reduction in the treatment of DDH patients relies on safe early concentric relocation of the dislocated femoral head. ${ }^{4}$ Closed reduction was accepted when the femoral head was seemed to be stable and, the ossific nucleus of the femoral head, if present, was seen medial to Perkin's line. 5 Soft tissue obstacles that may prevent concentric reduction of a dislocated hip includes inverted labrum, hypertrophied ligamentum teres, the capsule constriction by the iliopsoas tendon causing hourglass constriction over the acetabular introitus, hypertrophy and shortening of the transverse acetabular ligament, and the 
hypertrophied intra-articular fibrofatty tissue named pulvinar. ${ }^{6}$ The role of arthrography in the treatment of $\mathrm{DDH}$ is well established, its use is not universal. Arthrography has the advantages of viewing the shape and size of the cartilaginous part of both the femoral head and acetabulum, besides the soft tissue obstacles, which are obviously seen before reduction while the femoral head is dislocated. Arthrography also helps in the accurate assessment of the concentricity of hip reduction and the precise measurement of lateralization of the femoral head. Therefore, it assists in the prediction of potential treatment failures and complications. ${ }^{3,8}$ The aim of this work was to assess the role of arthrogram in evaluation of closed reduction of developmental dysplasia of the hip and to predict the requirements for open reduction in the presence of soft tissue obstacles.

\section{METHODS}

This prospective study included 30 patients with 36 involved hips who presented with DDH and planned for closed reduction. They were treated at EL-Hadra Orthopedic University hospital, during period between March 2011 and September 2015. An informed consent was taken from all patients, parents or guardians and after the approval of the local ethical committee of the university institution.

In this study, the safe zone in 10 hips (27.8\%) was relatively wide and the reduction was considered stable and in these cases no adductor tenotomy was done. On the other hand, in 26 hips $(72.2 \%)$ wide abduction or more than 10 or 15 degrees of internal rotation was required to maintain reduction, the reduction was considered unstable, and in these cases an adductor tenotomy was done to increase the safe zone by allowing a wider range of abduction. Arthrogram was done under general anesthesia, the patient was placed supine with the hips abducted 30-60 degrees and externally rotated, the symphysis pubis and the tendon of the adductor longus were palpated. Under image intensification, a 20-22gauge short bevel spinal needle was inserted at the lower border of the adductor longus tendon, 2-4 $\mathrm{cm}$ caudal to the inferior pubic symphysis (depending on the size of the child). The needle was inserted and directed towards the anterior superior iliac spine and should be remained parallel to the table. Fluoroscopy was used to confirm that the position of the needle tip is near the inferomedial aspect of the femoral head, after that the needle tip was introduced into the joint capsule. We injected a test dose of one $\mathrm{ml}$ of contrast and checked it by the fluoroscopy. In this study we used Urografin $76 \%$ (contains $100 \mathrm{mg}$ of sodium diatrizoate and $660 \mathrm{mg}$ of meglumine diatrizoate per $\mathrm{ml}$ ). The best concentration used was $3 \mathrm{ml}$ Urografin $76 \%$ diluted in $7 \mathrm{ml}$ saline and $3-5 \mathrm{ml}$ of diluted Urografin was injected through the needle into the joint. The needle was withdrawn followed by repeated passive motion of the hip distributing the contrast within the joint and over the surface of the femoral head. Arthrogram of normal side also done for comparison, and while the hip unreduced $X$ ray image was taken. Then the hip was gently reduced into a stable position, and a second $\mathrm{X}$ ray image was taken (Figure 1).
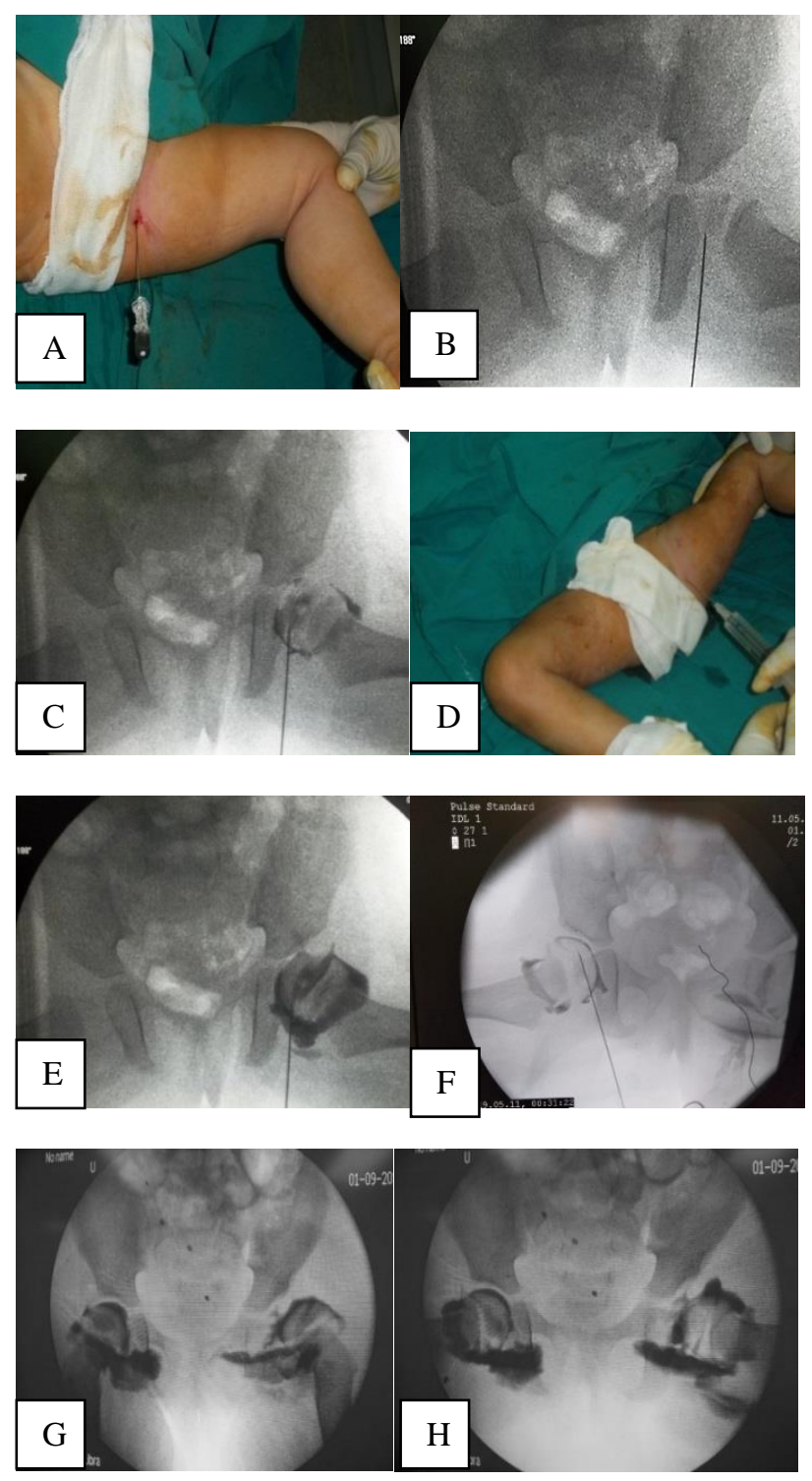

Figure 1: Technique or arthrogram in DDH- (A) 2022-gauge short bevel spinal needle inserted at the lower border of the adductor longus tendon and directed towards the anterior superior iliac spine; (B)

fluoroscopy used to confirm that the position of the needle tip is near the inferomedial aspect of the

femoral head; $(C, D)$ a test dose of one $\mathrm{ml}$ of contrast injected into the joint; (E) 3 to $5 \mathrm{ml}$ of diluted

Urographin injected into the joint; (F) arthrogram of normal side also done for comparison; (G) AP view after reduction and $(\mathrm{H})$ frog lateral view after reduction.

\section{Method of assessment}

Arthrography is helpful in determining presence of ossific nucleus and its position whether central or eccentric, acetabular dysplasia, femoral head sphericity 
and the position of the femoral head. More than $50 \%$ lateralization of the femoral head on the anteroposterior (AP) view was defined as subluxation and less than $50 \%$ lateralization was defined as dysplasia. Moreover, size of medial pooling (dye column between the femoral head and acetabulum) was compared to the normal side and if it was present within the double size, it was considered small medial pooling, if more than double considered large medial pooling. The most important role of arthrography is to delineate the presence of intraarticular obstacles for achieving anatomic reduction including inverted labrum (IL), hypertrophied ligamentum teres (LT), large transverse acetabular ligament (TAL) and hourglass (HG) constriction of the joint capsule.

\section{Methods of statistical analysis}

Data were analyzed by using SPSS software (Statistical package for social science for personal computers, IBM, Armonk, New York) using Pearson Chi-square test and comparing means, qualitative data were described using numbers and percent. Quantitative data were expressed as mean $\pm \mathrm{SD}$ and statistical significance was set at $\mathrm{p}=0.05$.

\section{RESULTS}

The youngest patient was 9 months and the oldest was 20 months with a mean age of $15.92 \pm 2.68$ months. This study included 7 males $(23.3 \%)$ and 23 females $(76.7 \%)$. There were 6 patients with bilateral hip involvement $(20.0 \%)$ and 24 patients with unilateral involvement $(80.0 \%)$, the right side was involved in 10 hips $(33.3 \%)$ while the left side involved in 14 hips (46.7\%).

The ossific nucleus was present in 33 femoral heads $(91.66 \%)$, it was concentrically placed in 12 hips $(33.33 \%)$ and eccentrically placed in 21 hips $(58.33 \%)$ and absent in three hips $(8.33 \%)$ which had a direct impact on the calculation of the CE angle of Wiberg. The central point calculated from arthrograms was more centrally placed in the osteocartilaginous head than that determined from the ossific nucleus observed. As regards femoral head sphericity in 6 hips $(16.7 \%)$ of the 36 hips with DDH femoral head was not spherical but somewhat flattened to confirm the shape of the superior lateral acetabulum or to the wall of the ilium where it was lodged.

The following data could be interpreted from the arthrography (Figure 2).

\section{Medial pooling of contrast medium}

The medial pool was small in 25 hips $(69.4 \%)$ and was large in 11 hips (30.6\%) (Figure 2A, B).

\section{Shape of the labrum}

It was founded that 6 hips $(16.7 \%)$ presented with inverted labrum (IL) which hindered reduction while the others 30 hips $(83.3 \%)$ the labrum was not an obstacle to the reduction (Figure $2 \mathrm{C}$ ).
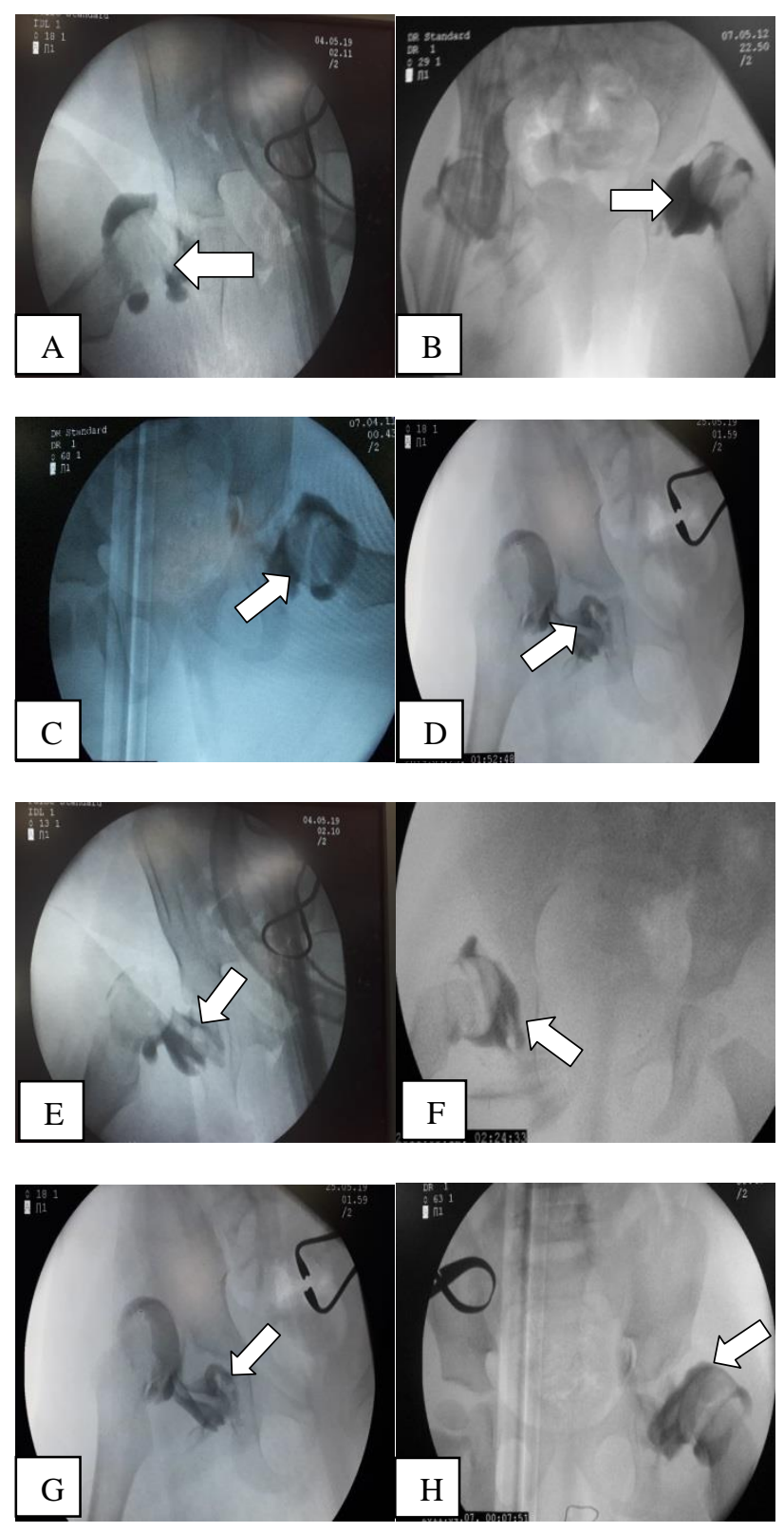

Figure 2: Different arthrographic findings (the arrows)- (A): small medial pool of contrast medium of right hip; (B): large medial pool of the left hip; (C): inverted labrum of the left hip; (D): hourglass constriction of the right hip; (E): hypertrophied ligamentum teres of the right hip; (F): large transverse acetabular ligament of the right hip; (G): dislocation of the right hip with hourglass constriction and hypertrophied inverted limbus and $(\mathbf{H})$ : subluxation of the left hip.

\section{Hourglass constriction}

An hourglass (HG) constriction was seen on the arthrogram of 5 hips $(13.9 \%)$ but the other 31 hips $(86.1 \%)$ did not show any constriction (Figure $2 \mathrm{D})$. 


\section{Ligamentum teres}

The ligamentum teres (LT) was observed to be enlarged and was a significant obstacle to reduction in 10 hips $(27.8 \%)$. In the other 26 hips $(72.2 .9 \%)$ it seemed to be normal (Figure 2E).

\section{Transverse acetabular ligament}

Seven hips (19.4\%) were shown to had large transvere acetabular ligament (TAL) while the other 29 hips $(80.6 \%)$ had normal transverse acetabular ligament (Figure 2F).
Out of the 36 hips, closed reduction was successful in 33 hips $(91.7 \%)$ and failed in $3(8.3 \%)$. After the interpretation of the arthrogram we considered the reduction of 7 out of the $33(21.2 \%)$ reduced hips nonconcentric because of the soft tissue obstacles to the reduction namely; hypertrophied LT in 7, HG constriction in 2 hips, large TAL in 4 and IL in 6 . Two out of 7 hips $(28.6 \%)$ showed the four obstacles, 2 (28.6\%) with hypertrophied LT, large TAL and IL, two (28.6\%) with IL and hypertrophied LT and one (14.3\%) with hypertrophied LT only. In the $3(8.3 \%)$ hips with failed closed reduction there were hypertrophied LT and HG constriction and large TAL in the all 3 hips.

Table 1: Demographic and clinical findings of the study cases.

\begin{tabular}{|c|c|c|}
\hline \multicolumn{2}{|l|}{ Variable } & Mean (range) or $\mathbf{N}(\%)$ \\
\hline \multicolumn{2}{|l|}{ Age (months) } & $15.92 \pm 2.86(9-20)$ \\
\hline \multirow{2}{*}{ Sex } & Male & $7(23.3)$ \\
\hline & Female & $23(76.7)$ \\
\hline \multirow{3}{*}{ Side } & Right & $10(33.3)$ \\
\hline & Left & $14(46.7)$ \\
\hline & Bilateral & $6(20.0)$ \\
\hline \multicolumn{2}{|l|}{ Acetabular index } & $40.72 \pm 5.45(34-55)$ \\
\hline \multirow{2}{*}{ Shape of the labrum } & Normal & $30(83.3)$ \\
\hline & Inverted & $6(16.7)$ \\
\hline \multirow{2}{*}{ Medial pooling } & Large & $11(30.6)$ \\
\hline & Small & $25(69.4)$ \\
\hline \multirow{2}{*}{ Hourglass constriction } & Absent & $31(86.1)$ \\
\hline & Present & $5(13.9)$ \\
\hline \multirow{3}{*}{ Ossific nucleus } & Absent & $3(8.3)$ \\
\hline & Concentric & $12(33.3)$ \\
\hline & Eccentric & $21(58.3)$ \\
\hline \multicolumn{2}{|l|}{ Follow up period (months) } & $32.56 \pm 6.06(24-48)$ \\
\hline \multirow{2}{*}{ Ligamentum teres } & Normal & $26(72.2)$ \\
\hline & Hypertrophied & $10(27.8)$ \\
\hline \multirow{2}{*}{$\begin{array}{l}\text { Transverse acetabular } \\
\text { ligament }\end{array}$} & Normal & $29(80.6)$ \\
\hline & Large & $7(19.4)$ \\
\hline \multirow{2}{*}{ Head sphericity } & Spherical & $30(83.3)$ \\
\hline & Non spherical & $6(16.7)$ \\
\hline \multirow{2}{*}{ Failure of CR } & No & $33(91.7)$ \\
\hline & Yes & $3(8.3)$ \\
\hline \multirow{2}{*}{ Medial OR } & No & $26(72.2)$ \\
\hline & Yes & $10(27.8)$ \\
\hline \multirow{4}{*}{$\begin{array}{l}\text { Soft tissue obstacles to } \\
\text { concentric closed reduction }\end{array}$} & $\begin{array}{l}\text { Hypertrophied LT, IL, large TAL and HG } \\
\text { constriction }\end{array}$ & 2/7 (28.57) \\
\hline & Hypertrophied LT, IL and lager TAL & 2/7 (28.57) \\
\hline & Hypertrophied LT and IL & $2 / 7(28.57)$ \\
\hline & Hypertrophied LT & $1 / 7(14.29)$ \\
\hline $\begin{array}{l}\text { Soft tissue obstacles in failed } \\
\text { closed reduction }\end{array}$ & Hypertrophied LT, large TAL and HG constriction & $3 / 3(100 \%)$ \\
\hline
\end{tabular}

LT: ligamentum teres; IL: inverted labrum; TAL: transverse acetabular ligament; HG: hourglass.

The labrum was shown to be inverted but we could not comment on it after reduction due to failure of closed reduction of these hips. In the 7 hips with non-concentric reduction besides the 3 hips with failed closed reduction, (total 10 out of 36 hips, [27.8\%]) we proceeded to open reduction of the hip through medial approach removing the soft tissue obstacles with the achievement of concentric reduction in all (Table 1). 
At the end of follow up, all hips (100\%) showed concentric reduction without re-dislocation in any case.

\section{DISCUSSION}

The role of arthrography in the treatment of DDH is well established. ${ }^{7}$ It is generally accepted that a successful outcome of closed reduction in the treatment of DDH patients relies on safe early concentric relocation of the dislocated femoral head. ${ }^{4}$ Severin followed by Leveuf presented the earliest detected description of the pathological findings seen on hip arthrography in DDH. ${ }^{9,10}$ Soft tissue obstacles that may prevent concentric reduction of a dislocated hip are produced as a result of secondary adaptive changes. They include inverted labrum, hypertrophied ligamentum teres, the iliopsoas tendon constricting the capsule causing hourglass constriction over the acetabular introitus, hypertrophy and shortening of the transverse acetabular ligament, and the hypertrophied intra-articular fibrofatty tissue named pulvinar. ${ }^{6}$

The technique of hip arthrography has been rather extensively reviewed in the literature with all authors in agreement as to the value of general anesthesia and fluoroscopic control. The medial approach to the hip joint is preferable in all pathologic hip conditions because the anatomical changes of interest have regularly been situated in the superior and lateral aspects of the joint. ${ }^{11}$

The neutral and Von Rosen views with arthrography provide useful information to distinguish between a subluxated hip with intervening soft tissues and a dysplastic hip with an increase of femoral anteversion. In the latter case, a poor reduction is reflected by an increase in the width of the medial dye column, but it is improved by the abduction and internal rotation applied with the Von Rosen view. ${ }^{12}$

The following data were assessed on the arthrogram:

\section{Femoral head sphericity}

In this study, $6(16.7 \%)$ out of the 36 hips with DDH, the femoral head was not spherical while in the other 30 hips $(83.3 \%)$ the head was spherical. In study done by Ishii et al, preoperative arthrogram showed that dislocated femoral head was not spherical in 44 hips out of 51 hips treated by open reduction. ${ }^{8}$ But intraoperatively the femoral head sphericity was correlated with arthrography finding in 36 hips only. They thought that ligamentum teres might interfere with the apparent shape of the femoral head, giving the false impression of a deformed head.

\section{Inverted labrum}

In this study we found that 6 hips (16.7\%) presented with IL which hindered reduction while the other 30 hip $(83.3 \%)$ the labrum was not an obstacle to the reduction.
Drummond et al mentioned that the arthrographic observations concerning the state of the labrum were confirmed at surgery in all patients treated with open reduction. $^{12}$ Clearly, the Leveuf dislocation and subluxation patterns could be distinguished. Renshaw emphasized that inadequate reduction by closed methods results in failure, especially when an inverted limbus obstructs concentric reduction. ${ }^{7}$ The inverted limbus, especially the anterior part, will seldom evert after walking has begun, an AP arthrogram shows only the superior portion of the limbus and is insufficient to assess its overall state. Lateral arthrography allows better visualization of all soft-tissue abnormalities including the anterior and posterior limbus, a tight iliopsoas tendon, and fibrous adhesions around the posterior capsule. In study done by Khoshhal et al an inverted limbus was seen in all cases in which the $\mathrm{L}$ distance was more than 4 mm. ${ }^{13}$ No statistically significant difference in the occurrence of AVN was found between hips with normal labrum and those with everted limbus, but a statistically significant difference in relation to developing AVN was found while comparing hips with both normal and everted limbus to hips with inverted limbus.

\section{Ligamentum teres}

The LT was observed to be enlarged and was a significant obstacle to reduction in 10 hips $(27.8 \%)$. In the other 26 hips $(72.2 \%)$ it seemed to be normal. Reduction without arthrographic guidance depends exclusively on a feeling of stable reduction and on the surgeon impression of concentricity of the reduction relying on the position of the bony ossific nucleus of the femoral head (if present), which may also be misleading if the femoral head is deformed with an eccentric ossific nucleus.

Arthrogram traditionally has been used at the time of reduction and at the follow up examination to assess whether femoral head is centered within the acetabulum. Lonnerholm carried out repeat arthrography in 130 children within one month but did not address the significance of its use. ${ }^{14}$ Forlin et al reported that repeat arthrograms, when the cast was changed after reduction, did not provide prognostic information. ${ }^{15}$ Herring emphasized the importance of confirming reduction by arthrography in 59 hips treated with closed reduction. ${ }^{4}$ Their results confirm that the most important factor that determines outcome of closed treatment of congenital hip dislocation is the quality of the initial reduction. In hips with good or adequate reductions, $94 \%$ had good or acceptable results. Conversely, in hips with poor reductions and hips in which the quality of reduction could not be determined, only $21 \%$ had acceptable results. They also noted that as the quality of reduction declined, treatment time increased.

Other imaging techniques such as ultrasound, CT and MRI are being used increasingly instead of arthrography in the evaluation of hip dysplasia. Ultrasound that is non- 
invasive and dynamic used primarily in screening for DDH but lacks definition to determine the exact anatomic obstructions to hip reduction. ${ }^{16}$ Although ultrasound is helpful in diagnosing the dislocated hip in the infant, its application to surgical decision making is limited. CT is non-invasive but the high radiation dose of computerized tomography, its poor quantification and lacks resolution in evaluating cartilage and soft tissue, particularly in the sagittal and coronal reconstruction views make it of little value. ${ }^{17}$ Resolution of the soft tissues is excellent with magnetic resonance imaging, but young children may need an anaesthetic and only open scanners allow dynamic examination. This imaging cannot be done in the operative theatre in conjunction with surgical anesthesia. $^{18,19}$

Studer et al have performed their series on 38 hips in 29 patients underwent closed reduction for treatment of DDH. ${ }^{20}$ They correlated the intraoperative arthrogram findings with the post-reduction MRI identifying the obstacles to complete reduction. They concluded that the main obstacles identified on MRI were the LT (15.8\%), labrum $(13.1 \%)$ and acetabular roof cartilage hypertrophy $(13.2 \%)$.

In this study, 7 out of 36 hips showed nonconcentric reduction with large medial pooling besides one or more soft tissue obstacles. Although they appeared reduced in $\mathrm{X}$-ray imaging. We relied on arthrogram in these findings and proceed to open reduction through medial approach with clearing of the acetabulum out of any soft tissues preventing concentric reduction. Relaying on arthrogram to proceed to open reduction achieved an excellent result at the end of follow up without re-dislocation or avascular necrosis of the head of the femur in any of the studied cases.

Ishii et al have shown that there is a reliable correlation between the observations made at arthrography and those made at open reduction. ${ }^{8}$ Arthrography proved effective in identifying the concentricity of reduction (medial dye column), the configuration of the osteocartilagenous structures, and the presence of soft tissue obstacles (limbus, and LT). Gans et al showed a positive correlation between intraoperative arthrography and the anatomic position of the femoral head shown by MRI. They suggested that a MDP of $\leq 6 \%$ of femoral head width may be a suitable intraoperative criterion for proving the adequacy of $\mathrm{CR} .^{21}$

Arthrography proved reliable in outlining the inverted limbus that can obstruct closed reduction. Because the femoral head ossification center was frequently eccentric, the center edge angle of Wiberg was calculated from arthrography more accurately than from radiograms. In a study done by Kontis et al, they were obliged to modify the preoperative plans in $57 \%$ (12 of 21) of patient with DDH after performing arthrogram. ${ }^{22}$
Zhang et al stated in their study that the arthrography has an important role in predicting residual hip dysplasia and they concluded that more than $80 \%$ of hips femoral head coverage of $<30 \%$ calculated by intraoperative arthrography have persistent hip dysplasia and may accordingly need open reduction to attain better hip reduction. $^{23}$

\section{Complications}

In this study no reliable complication occurred regardless the dye extravasation happened in some cases that directed along the sheath of either the iliopsoas or adductor muscle and did not interfere with diagnostic quality of the examination, since contrast in no way overlies the hip joint. The radiopaque contrast media solutions used have a large margin of safety with a very low incidence of side effects.

\section{CONCLUSION}

It could be concluded that hip arthrogram is a very reliable method in diagnosing hip concentricity and the presence of soft tissue obstacles in management of developmental hip dysplasia by closed reduction. Arthrogram helps the operator to decide open reduction in such cases achieving excellent results in all cases.

Funding: No funding sources

Conflict of interest: None declared

Ethical approval: The study was approved by the institutional ethics committee

\section{REFERENCES}

1. Beaty H. Congenital and developmental dysplasia of the hip. In: Canale T, ed. Cambell's Operative Orthopaedics. 9th ed. St Louis, Boston, Chicago, London, Toronto, Baltimore: CV Mosby Company; 1998: 1079-1117.

2. Hadlow V. Neonatal screening for congenital dislocation of the hip. A prospective 21-year survey. J Bone Joint Surg (Br). 1988;70(5):740-3.

3. Jaglan SS, Crawford AH, Dias LS, Roy DR, Tachdjian MO. Closed versus open reduction of congenital dislocation of the hip in children between six and twenty-four months of age. Orthop Trans. 1991;15:736-41.

4. Race C, Herring JA. Congenital dislocation of the hip. An evaluation of closed reduction. J Pediatr Orthop. 1983;3:166-72.

5. Ali B, Haluk A, Hakan O. The effect of arthrographically detected femoral head lateralization and soft tissue interposition during closed reduction of developmental dislocation of the hip on mid-term results. Acta Orthop Traumatol Turc. 2004;38(1):1-7.

6. Wenstien SL, Morrissy RT. Natural history of congenital hip dislocation and hip dysplasia. 4th ed. 
Lovell and Winter's Pediatric Orthopedics. 2000: 984-1013.

7. Renshaw TS. Inadequate reduction of congenital dislocation of the hip. J Bone Joint Sur (Am). 1981;63:1114-21.

8. Ishii, Weinstien SL, Ponseti IV. Correlation between arthrogram and operative finding in congenital dislocation of the hip. Clin Orthop. 1980;153:138-45.

9. Severin E. Arthrography in congenital dislocation of the hip. J Bone Joint Surg (Am). 1939;21(2):304-13.

10. Leveuf J. Primary congenital subluxation of the hip. J Bone Joint Surg (Am). 1947;29:149-62.

11. Alan MS, Michael JG. Hip arthrography in children. Skeletal Radiol. 1978;3:155-60.

12. Dennis SD, Johno D, Alanb R, Albert MJ, Robertsonm WW. Arthroraphy in the evaluation of congenital dislocation of the hip. Clin Ortho. 1989;243:148-56.

13. Khoshhal KI, Kremli MK, Zamzam MM, Akod OM. The role of arthrography guided closed reduction in minimizing the incidence of avascular necrosis in developmental dysplasia of the hip. $\mathrm{J}$ Pediatr Orthp. 2005;14(4):256-61.

14. Lonnerholm T. Arthography of the hip in children. Acta Radiol Diag (Stockh). 1980;21(2):279-92.

15. Forlin E, Choi IH, Guille JT, Bowen JR, Glutting J. Prognostic factors in congenital dislocation of the hip treated with closed reduction. The importance of arthrographic evaluation. J Bone Joint Surg (Am). 1992;74:1140-52.

16. Bar E, Meyer S, Harari G, Port S. Ultrasonography of the hip in developmental hip dysplasia. J Bone Joint Surg (Br). 1998;80(2):321-4.
17. Hernandez RJ. Concentric reduction of the dislocated hip: Computed-tomographic evaluation. Radiol. 1984;150:266.

18. Laor T, Dennis R, Charles T, Mehlman DO. Limited magnetic resonance imaging examination after surgical reduction of developmental dysplasia of the hip. J Pediatr Orthop. 2000;20:572-4.

19. Suzuki S, Kashiwagi N, Seto Y, Muki S. Location of the femoral head in developmental dysplasia of the hip: three-dimensional evaluation by means of magnetic resonance image. J Pediatr Orthop. 1999;19:88-91.

20. Studer K, Williams N, Studer P, Baker M, Glynn A, Foster BK, et al. Obstacles to reduction in in $\neg$ fantile developmental dysplasia of the hip. J Child Orthop. 2017;11:358-66.

21. Gans I, Sankar WN. The medial dye pool revisited: correlation between arthrography and MRI in closed reductions for DDH. J Pediatr Orthop. 2014;34:78790.

22. Kotnis R, Spiteri V, Little C, Theologis T, Wainwright A, Benson MK. Hip arthrography in the assessment of children with developmental dysplasia of the hip and Perthes' disease. J Pediatr Orthop B. 2008;17(3):114-9.

23. Zhang Z, Zhe Fu, Yang J, Wang K, Xie L, Deng S, et al. Intraoperative arthrogram predicts residual dysplasia after successful closed reduction of DDH. Orthopaedic Surg. 2016;8(3):338-44.

Cite this article as: Ahmed AA, Fadel MED. Role of intraoperative arthrogram in decision making of closed versus medial open reduction of developmental hip dysplasia. Int J Res Orthop 2019;5:1037-43. 